\title{
Decreased T1 Contrast between Gray Matter and Normal-Appearing White Matter in CADASIL
}

\author{
F. De Guio, S. Reyes, M. Duering, L. Pirpamer, H. Chabriat, and E. Jouvent
}

\begin{abstract}
BACKGROUND AND PURPOSE: CADASIL is the most frequent hereditary small-vessel disease of the brain. The clinical impact of various MR imaging markers has been repeatedly studied in this disorder, but alterations of contrast between gray matter and normal-appearing white matter remain unknown. The aim of this study was to evaluate the contrast alterations between gray matter and normal-appearing white matter on T1-weighted images in patients with CADASIL compared with healthy subjects.
\end{abstract}

MATERIALS AND METHODS: Contrast between gray matter and normal-appearing white matter was assessed by using histogram analyses of 3D T1 high-resolution MR imaging in 23 patients with CADASIL at the initial stage of the disease (Mini-Mental State Examination score $>24$ and modified Rankin scale score $\leq 1$; mean age, $53.5 \pm 11.1$ years) and 30 age- and sex-matched controls.

RESULTS: T1 contrast between gray matter and normal-appearing white matter was significantly reduced in patients compared with age- and sex-matched controls (patients: $1.35 \pm 0.08$ versus controls: $1.43 \pm 0.04, P<10^{-5}$ ). This reduction was mainly driven by a signal decrease in normal-appearing white matter. Contrast loss was strongly related to the volume of white matter hyperintensities.

CONCLUSIONS: Conventional 3D T1 imaging shows significant loss of contrast between gray matter and normal-appearing white matter in CADASIL. This probably reflects tissue changes in normal-appearing white matter outside signal abnormalities on T2 or FLAIR sequences. These contrast alterations should be taken into account for image interpretation and postprocessing.

ABBREVIATIONS: CADASIL = cerebral autosomal dominant arteriopathy with subcortical infarcts and leukoencephalopathy; NAWM = normal-appearing white matter; MMSE $=$ Mini-Mental State Examination

$C$ erebral autosomal dominant arteriopathy with subcortical infarcts and leukoencephalopathy (CADASIL) is a hereditary small-vessel disease of the brain secondary to mutations of the NOTCH3 gene. ${ }^{1}$ Conventional MR imaging markers have been repeatedly investigated in this disorder. ${ }^{2-5}$ The impact of lacunar lesions detected on T1-weighted sequences seems more impor-

Received January 15, 2013; accepted after revision April 4.

From University Paris Diderot (F.D.G., H.C., E.J.), Sorbonne Paris Cité, Paris, France; Department of Neurology (S.R., H.C., E.J.), Lariboisière Hospital, Paris, France; Institute for Stroke and Dementia Research (M.D.), Medical Centre, Klinikum der Universität München, Ludwig-Maximilians-University, Munich, Germany; and Division of Neurogeriatrics (L.P.), Department of Neurology, Medical University of Graz, Graz, Austria.

This work was supported by grants from the Fondation Planiol, the Fondation NRJInstitut de France, and an FP6 ERANET NEURON (01 EW1207) and was helped by the French CADASIL Foundation.

Please address correspondence to Eric Jouvent, MD, Hôpital Lariboisière, 2 Rue Ambroise Paré, 75010 Paris, France; e-mail: eric.jouvent@lrb.aphp.fr

-- Indicates open access to non-subscribers at www.ajnr.org

http://dx.doi.org/10.3174/ajnr.A3639 tant than that of white matter lesions observed on FLAIR sequences. ${ }^{6}$ Recently, various measures of brain and cortical atrophy were shown to be related to clinical worsening. ${ }^{7,8}$

As reported in the context of Alzheimer disease, ${ }^{9}$ contrast between gray matter and normal-appearing white matter (NAWM) may be altered in CADASIL. This could have important implications for both image interpretation in the clinical setting and postprocessing in research studies. So far however, the alterations of MR imaging T1 contrast between GM and NAWM have not been evaluated in CADASIL. The aim of the present study was to assess potential contrast alterations between GM and NAWM on T1weighted images in patients with CADASIL at the initial stage of the disease compared with age- and sex-matched individuals.

\section{MATERIALS AND METHODS \\ Participants}

Patients with CADASIL without dementia (Mini-Mental State Examination [MMSE] score $>24)^{10}$ and without significant disability (modified Rankin Scale score $\leq 1)^{6}$ were recruited from 


\begin{tabular}{|c|c|c|c|}
\hline & Patients with CADASIL $(n=22)$ & Healthy Controls $(n=29)$ & $P$ Value \\
\hline Age (yr) (mean) (range) & $53.5 \pm 11.1,32.1-74.5$ & $53.8 \pm 11.0,30.1-71.4$ & .73 \\
\hline Male sex (No.) (\%) & $11 / 22(50 \%)$ & $15 / 29(52 \%)$ & .88 \\
\hline Right-handed (No.) (\%) & $22 / 22(100 \%)$ & $28 / 29(97 \%)$ & .99 \\
\hline Level of education (yr) & $11.7 \pm 3.9$ & $13.6 \pm 3.5$ & .08 \\
\hline MMSE (mean) (median) (range) & $28.5,29,25-30$ & $29.0,29,26-30$ & .26 \\
\hline Brain volume in $\mathrm{mm}^{3}$ (mean) & $1017202 \pm 113233$ & $1003640 \pm 84340$ & $.64(.61)^{\mathrm{a}}$ \\
\hline Normalized GM peak (mean) & $0.30 \pm 0.05$ & $0.31 \pm 0.05$ & $.48(.45)^{\mathrm{a}}$ \\
\hline Normalized WM peak (mean) & $0.59 \pm 0.06$ & $0.67 \pm 0.09$ & $.001(.001)^{\mathrm{a}}$ \\
\hline Contrast between GM and NAWM (mean) & $1.35 \pm 0.08$ & $1.43 \pm 0.04$ & $.001\left(<10^{-5}\right)^{a}$ \\
\hline White matter lesion volume, (mean) (median) (range) in $\mathrm{mm}^{3}$ & $97972.9,76256.6,7249.8-267095.1$ & NA & - \\
\hline Lacunar lesion volume (mean) (median) (range) in $\mathrm{mm}^{3}(n=14 / 22,64 \%)^{\mathrm{b}}$ & 526.1, 290.1, 14.0-1974.7 & NA & - \\
\hline No. of microhemorrhages (mean) (median) (range) $(n=8 / 22,36 \%)^{\mathrm{b}}$ & $6.5,2.5,1-32$ & NA & - \\
\hline
\end{tabular}

Note:-NA indicates not applicable; -, no test performed.

${ }^{a} P$ value adjusted for age and sex.

${ }^{b}$ In patients with such lesions (number given in parentheses)

our French data base on a voluntary basis. Twenty-three patients were included. Thirty healthy controls from a local volunteer data base were age- and sex-matched. A local ethics committee validated the protocol.

\section{Clinical Assessment}

All participants underwent a comprehensive neuropsychological evaluation preceded by a clinical psychological interview. Global cognitive performances were assessed by using the MMSE. ${ }^{10}$ This widely used global cognitive battery scores from 0 to 30 . As a general rule, patients with a score under 24 are considered to have dementia. ${ }^{11}$ Disability was assessed by using the modified version of the Rankin Scale (from 0, no disability, to 5, major disability), in which scores $\leq 1$ denote no disability. ${ }^{6}$ Other tests, including those of visual object or spatial perception and verbal or visual episodic memory and the evaluation of attention and executive processes, were performed but are not detailed here because they were not relevant to the present study.

\section{MR Imaging Protocol}

3D T1 images were acquired with a $3 \mathrm{~T}$ Tim Trio MR imaging scanner (Siemens, Erlangen, Germany) equipped with a 12-channel head coil, by using a single standard MPRAGE sequence (inplane resolution $=1 \times 1 \mathrm{~mm}^{2}$, section thickness $=1.1 \mathrm{~mm}, \mathrm{TR}=$ $2300 \mathrm{~ms}, \mathrm{TE}=2.98 \mathrm{~ms}$, TI $=900 \mathrm{~ms}$, flip angle $\left.=9^{\circ}\right)$. Within 6 months, as part of another ongoing longitudinal study, patients also underwent an MR imaging protocol on a 1.5T Signa scanner (GE Healthcare, Milwaukee, Wisconsin) with 3D T1 fast-spoiled gradient echo (in-plane resolution $=1.02 \times 1.02 \mathrm{~mm}^{2}$, section thickness $=0.8 \mathrm{~mm}, \mathrm{TR}=9 \mathrm{~ms}, \mathrm{TE}=2 \mathrm{~ms}$, flip angle $=20^{\circ}$ ), FLAIR (in-plane resolution $=1 \times 1 \mathrm{~mm}^{2}$, section thickness $=5.5$ $\mathrm{mm}, \mathrm{TR}=8402 \mathrm{~ms}, \mathrm{TE}=161 \mathrm{~ms}, \mathrm{TI}=2002 \mathrm{~ms}$ ), and $\mathrm{T} 2 *$ gradient echo (in-plane resolution $=1 \times 1 \mathrm{~mm}^{2}$, section thickness $=5.5 \mathrm{~mm}, \mathrm{TR}=500 \mathrm{~ms}, \mathrm{TE}=15 \mathrm{~ms}$ ) sequences, from which lesion masks, volumes of lacunar lesions and of white matter hyperintensities, and the number of cerebral microhemorrhages were obtained as previously reported. ${ }^{6,7}$

\section{Image Processing}

3D T1-weighted images were segmented to obtain brain masks for GM and WM by using the Morphologist pipeline of BrainVISA (http://www.brainvisa.info), which relies on a histogram analysis algorithm performed after a first step consisting of field inhomo- geneity bias correction. ${ }^{12}$ Lacunar lesions were defined on T1weighted images as hypointense lesions with a signal identical to that of CSF, a diameter of $>3 \mathrm{~mm}$, and a distinct shape from that of enlarged perivascular spaces. ${ }^{13}$ Microhemorrhages were defined as rounded foci of signal loss with a diameter inferior to 5 $\mathrm{mm}$ on gradient-echo sequences and distinct from vascular flow voids, leptomeningeal hemosiderosis, or nonhemorrhagic mineralization. Masks of white matter hyperintensities were obtained from FLAIR sequences by applying an adaptive threshold derived from the signal-intensity histogram and were manually corrected afterward only if necessary, as previously detailed. ${ }^{14}$ Registration matrices between FLAIR and 3D T1 sequences were calculated with the FLIRT rigid body registration algorithm (www.fmrib.ox. ac.uk/fsl) and then were applied on masks of white matter hyperintensities by using nearest neighbor interpolation to obtain binary masks of white matter lesions in the space of the 3D T1 sequence, as previously reported. ${ }^{7}$ In patients, histograms of biascorrected 3D T1 images were computed within the brain mask and outside the lesion mask to study GM and NAWM. Histograms were computed on whole GM and WM in control subjects. To ensure that computing histograms in smaller and/or different volumes did not systematically bias our results, we checked analyses after random attribution of lesion masks of patients to MR imaging scans of control subjects. The contrast between GM and NAWM was defined as the ratio of the intensity level corresponding to the NAWM peak and the one corresponding to the GM peak. Image intensity was normalized between subjects according to their minimum values (representing at least 1000 voxels) and to the intensity level of the vitamin E capsule that was placed before MR imaging acquisition to indicate the right side of the subject.

\section{Statistical Methods}

R software (http://www.R-project.org) was used for statistical analyses. Group comparisons were made according to variable types and distributions. For numeric variables, we used $t$ tests when variables were normally distributed, taking into account the equal or unequal variances of the 2 groups. When the assumption of normality was violated, the Wilcoxon rank sum tests were used. For categoric variables, $\chi^{2}$ tests were used. Contrast differences between groups were adjusted for age and sex, which may influence tissue contrast, ${ }^{15}$ by using ANCOVA. 


\section{6-year-old male control}
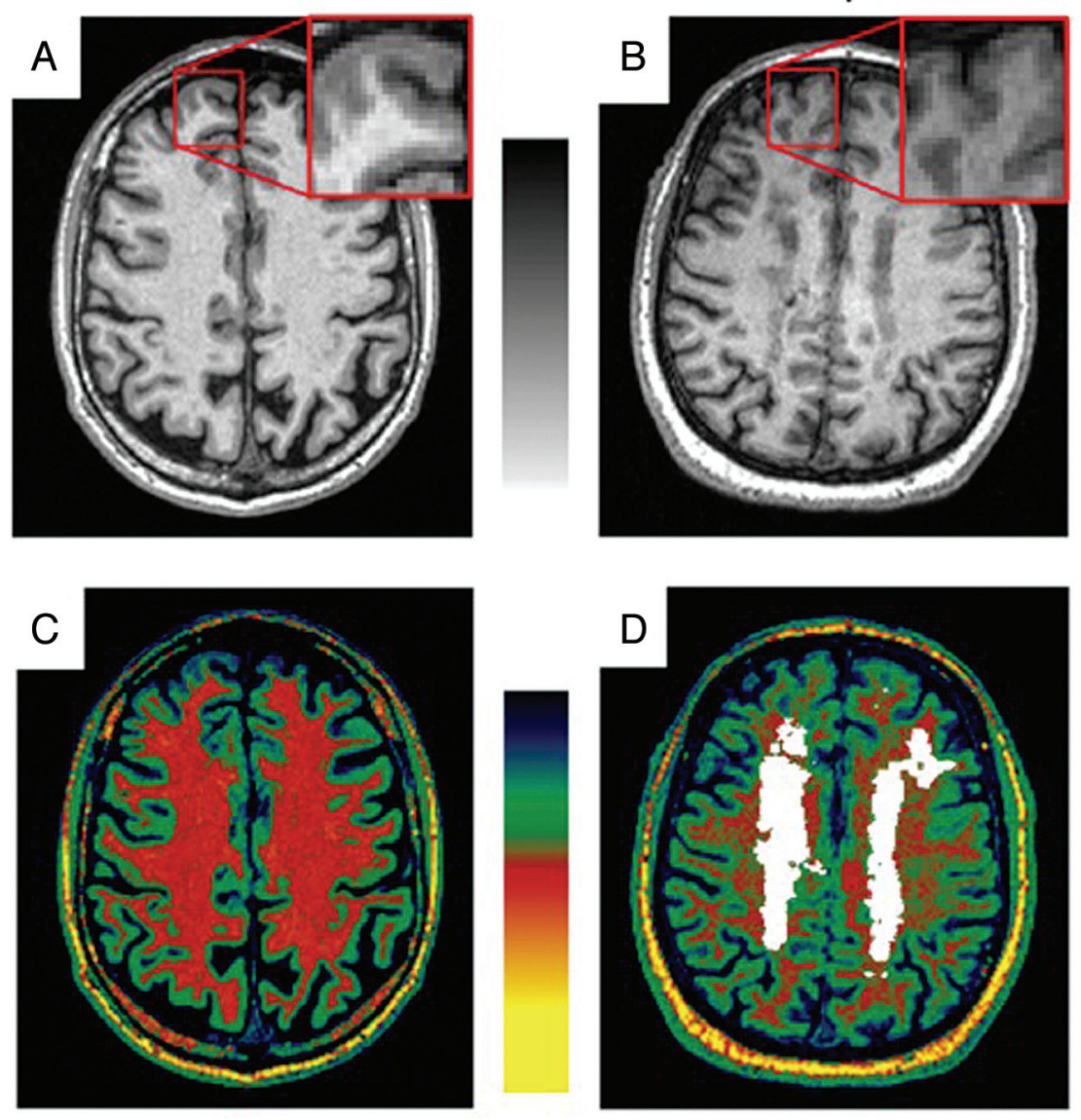

FIG 1. Reduction of $\mathrm{Tl}$ contrast between gray matter and normal-appearing white matter. Axial 3D T1 image after intensity normalization of a 56-year-old healthy male subject $(A)$ and a 56-yearold male patient with CADASIL $(B)$ with zoom boxes showing reduced contrast between gray matter and normal-appearing white matter in CADASIL because NAWM appears darker. Colorcoded images are enhancing the global heterogeneity of NAWM outside the lesions determined on FLAIR (overlaid in white) in the patient with CADASIL (D) compared with the homogeneous white matter in the control subject $(C)$. The signal of subcutaneous fat, similar for the 2 subjects, may serve as a reference for visual inspection.

\section{RESULTS}

One control subject did not undergo MR imaging, and histogram analysis failed for 1 patient with CADASIL due to a single peak detection, leaving 22 patients and 29 control subjects for analyses. Characteristics of the 2 groups are reported in the Table. Age, sex, level of education, MMSE score, and brain volumes did not significantly differ between patients and controls.

We observed a significant contrast reduction between GM and NAWM in patients compared with controls, which persisted after adjustment for age and sex as illustrated in Fig 1 (patients: $1.35 \pm$ 0.08 versus controls: $1.43 \pm 0.04, P<10^{-5}$ ). These contrast alterations were mainly driven by modifications of NAWM because normalized values showed a decreased NAWM peak in patients (patients: $0.59 \pm 0.06$ versus controls: $0.67 \pm 0.09, P=.001$ ) but no difference in GM peaks (Fig 2).

Applying white matter lesion masks of patients on scans of control subjects randomly did not alter the NAWM peak computation (unchanged in 14 subjects, mean variation of $0.2 \%$ in the other 8 subjects).
In patients, linear regression modeling showed that contrast between GM and NAWM was strongly related to the logarithm of white matter lesion volume after adjustment for age and sex (estimate: -0.09 ; SE: $0.01 ; P<10^{-4}$ ). The contrast was not related to the volume of lacunar lesions (estimate: -4.97 ; SE: $2.99 ; P=.12$ ) or to the number of microhemorrhages (estimate: -0.002 ; SE: 0.003; $P=.38)$.

In patients, we also observed a marginal linear relationship between MMSE scores and contrast between GM and NAWM after adjustment for age and sex (estimate: 9.6; SE: 4.4; $P$ value $=.04$ ). No other association with other clinical outcomes was significant.

\section{DISCUSSION}

In the present study, we observed significant contrast alterations between NAWM and GM in patients with CADASIL compared with age- and sexmatched healthy subjects, long before the appearance of disability. Our results seemed mainly related to a global reduction of NAWM T1 signal in patients with CADASIL. These findings are in line with previous data obtained in CADASIL that showed alterations of the NAWM by using magnetization transfer $^{16}$ or diffusion tensor imaging. ${ }^{17}$ In contrast to these sophisticated methods, our results were obtained on conventional T1-weighted images by using simple postprocessing steps and were easily confirmed by visual inspection of patient scans compared with controls (Fig 1).

The decreased signal in NAWM observed in patients with CADASIL may be explained by diffuse white matter edema as recently reported. ${ }^{18}$ An increase in water content would induce longer T1 and thus reduce signal in T1-weighted images. Demyelination, neuronal degeneration, or regional iron concentration may also be involved in these signal changes. ${ }^{19}$

Contrast reduction was previously observed in normal aging ${ }^{20}$ and was proposed as an alternative marker of tissue loss in Alzheimer disease. ${ }^{9}$ Here, the contrast reduction was strongly and independently related to the extent of WM lesions in patients. A correlation between average magnetization transfer imaging metrics of the NAWM and lesion volume was previously reported in CADASIL, which authors attributed to Wallerian degeneration secondary to axonal injury. ${ }^{16}$

Our results may have consequences for postprocessing of 3D T1 images in research studies. Indeed, high-resolution 3D T1 acquisitions are necessary so far to perform fine-tissue segmentation, voxel-based morphometry studies, and advanced image techniques such as cortical surface analysis ${ }^{7}$ or cortical 


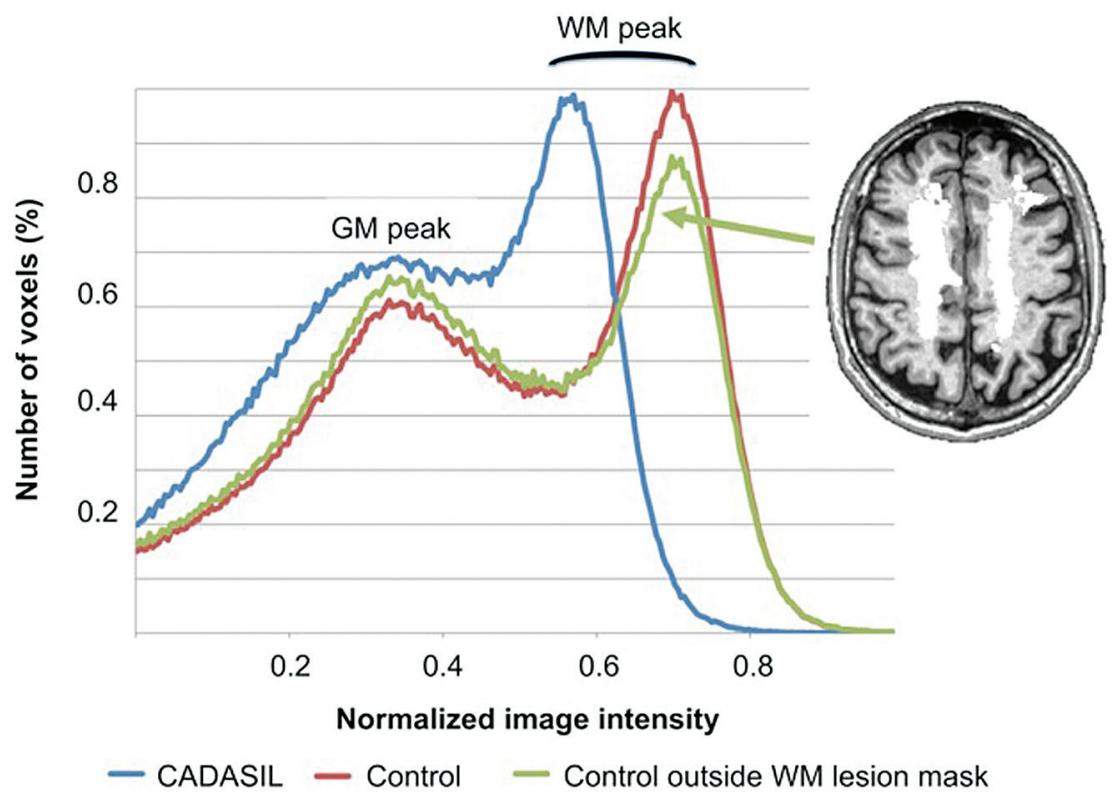

FIG 2. $3 D T 1$ intensity histograms in a patient with CADASIL and a control subject. Normalized histograms computed within the brain and outside the white matter lesion masks for the 56-yearold patient with CADASIL and control subject of Fig 1. For the control subject, a histogram is redrawn after exclusion of voxels corresponding to the white matter lesion mask of the patient (overlaid in white). While the gray matter peak is roughly the same between subjects, the white matter peak is left-shifted in the patient with CADASIL, leading to a reduced contrast between GM and NAWM. Computation of the histogram for the control subject outside the lesion mask (randomly allocated from a patient with CADASIL) did not change WM peak and contrast values.

thickness estimation ${ }^{8}$ through dedicated software, and it has been demonstrated that contrast properties could influence cortical thickness measures. ${ }^{9,21}$ Contrast alterations may also serve as a visual clue to the diagnosis of CADASIL because they do not seem to be as severe in other types of small-vessel disease of the brain, but further studies will be necessary to evaluate this hypothesis.

We observed a significant relationship between MMSE scores and contrast changes in patients with CADASIL. However, this finding should be interpreted cautiously because MMSE scores lay within normal ranges and the $P$ value was marginally significant (.04). Moreover, we did not observe any other association between cognitive tests and contrast changes in patients. Further studies with larger samples and patients with more severe disease will help to determine the clinical significance of $\mathrm{T} 1$ contrast changes.

Our study has some limitations. FLAIR sequences were not part of the $3 \mathrm{~T}$ protocol because it was designed for a cortical morphology study. Control subjects had no lesion mask, and histograms were computed on the whole WM. However, we showed that applying WM lesion masks of patients on control scans randomly did not change the results. Besides, at least some part of WM lesions are hypointense on T1-weighted images. Therefore, lesions potentially included in the WM would have induced a decreased contrast in controls, which is the opposite of what we found (ie, an increased contrast in controls). FLAIR sequences for patients were obtained within 6 months by using $1.5 \mathrm{~T}$ acquisitions and 5-mm-thick sequences. Hypointense lesions on T1 may thus appear outside lesion masks determined on FLAIR. However, the extension of white matter lesions in nondisabled patients with CADASIL in such a short period is negligible, unlikely to alter our results. ${ }^{7}$ Moreover, manual corrections performed to include potential hypointense lesions on T1 secondary to this time interval or to other potential sources such as variation of field strength, differences in section thickness between FLAIR and 3D-T1, or registration imprecisions and not overlaid by lesion masks did not alter our results (data not shown).

Another limitation of our study was the global computation of the contrast between GM and NAWM, not allowing a regional quantification of contrast reduction. This global measure was thought to be more robust, given the large variability of WM lesion extent in patients with CADASIL, and highlighted a diffuse biologic mechanism occurring in whole NAWM. Finally, the signal normalization chosen to compare NAWM mean intensity across subjects was not perfect because the vitamin $\mathrm{E}$ capsule was not equally placed on subjects and therefore was dependent on gradient nonlinearity effects and field heterogeneities. However, the large differences observed between the 2 groups in NAWM and not in GM could not be explained only by field heterogeneities.

Our study also has several strengths. There was a high homogeneity between groups in terms of age and sex, which was important, given the effect of age on contrast. Patients and controls were imaged with the same MR imaging scanner and protocol, with special care to reproduce acquisition conditions. The histogrambased technique to compute the tissue contrast is simple but was robust and adapted to patients with large WM lesions, leading to straightforward and original results.

\section{CONCLUSIONS}

Conventional 3D T1 imaging shows significant loss of contrast between GM and NAWM as soon as the early stages in CADASIL. These contrast alterations should be taken into account for both image interpretation and postprocessing. Whether these alterations can be used as a new biomarker in CADASIL or in other small-vessel diseases of the brain requires further investigation.

\section{ACKNOWLEDGMENTS}

The authors acknowledge Jocelyne Ruffié, Veronique Joly-Testaud, Laurence Laurier, and Chantal Ginisty for the recruitment process of patients and controls.

Disclosures: François De Guio-RELATED: Grant: ERA-NET-Funded Collaborative Network MESCOG, ${ }^{*}$ Comments: The network is funded under the ERA-Net scheme in FP6 2007-2011 by the European Commission. Marco Duering-UNRELATED: Grants/Grants Pending: BMBF/DZNE, Travel/Accommodations/Meeting Expenses Unrelated to Activities Listed: United Leukodystrophy Foundation. Hugues Chabriat-UNRELATED: Board Membership: Lundbeck, Janssen, Comments: steer- 
ing or safety committees, Consultancy: Lundbeck, Janssen, Comments: during the past 5 years. *Money paid to the institution.

\section{REFERENCES}

1. Chabriat H, Joutel A, Dichgans M, et al. CADASIL. Lancet Neurol 2009;8:643-53

2. Yousry TA, Seelos K, Mayer M, et al. Characteristic MR lesion pattern and correlation of $\mathrm{T} 1$ and $\mathrm{T} 2$ lesion volume with neurologic and neuropsychological findings in cerebral autosomal dominant arteriopathy with subcortical infarcts and leukoencephalopathy (CADASIL). AJNR Am J Neuroradiol 1999;20:91-100

3. Singhal S, Rich P, Markus HS. The spatial distribution of MR imaging abnormalities in cerebral autosomal dominant arteriopathy with subcortical infarcts and leukoencephalopathy and their relationship to age and clinical features. AJNR Am J Neuroradiol 2005;26:2481-87

4. van Den Boom R, Lesnik Oberstein SA, van Duinen SG, et al. Subcortical lacunar lesions: an MR imaging finding in patients with cerebral autosomal dominant arteriopathy with subcortical infarcts and leukoencephalopathy. Radiology 2002;224:791-96

5. Duering M, Zieren N, Herve D, et al. Strategic role of frontal white matter tracts in vascular cognitive impairment: a voxelbased lesion-symptom mapping study in CADASIL. Brain 2011;134:2366-75

6. Viswanathan $\mathrm{A}$, Godin $\mathrm{O}$, Jouvent $\mathrm{E}$, et al. Impact of MRI markers in subcortical vascular dementia: a multi-modal analysis in CADASIL. Neurobiol Aging 2010;31:1629-36

7. Jouvent E, Mangin JF, Duchesnay E, et al. Longitudinal changes of cortical morphology in CADASIL. Neurobiol Aging 2012;33:1002.e29-36

8. Duering M, Righart R, Csanadi E, et al. Incident subcortical infarcts induce focal thinning in connected cortical regions. Neurology 2012;79:2025-28

9. Grydeland H, Westlye LT, Walhovd KB, et al. Improved prediction of Alzheimer's disease with longitudinal white matter/gray matter contrast changes. Hum Brain Mapp 2013;34:1775-85

10. Folstein MF, Folstein SE, McHugh PR. "Mini-mental state": a prac- tical method for grading the cognitive state of patients for the clinician. J Psychiatr Res 1975;12:189-98

11. Jagust W, D'Esposito M. Imaging the Aging Brain. New York: Oxford University Press; 2009

12. Jouvent $\mathrm{E}$, Viswanathan $\mathrm{A}$, Mangin J-F, et al. Brain atrophy is related to lacunar lesions and tissue microstructural changes in CADASIL. Stroke 2007;38:1786-90

13. Zhu YC, Dufouil C, Mazoyer B, et al. Frequency and location of dilated Virchow-Robin spaces in elderly people: a populationbased 3D MR imaging study. AJNR Am J Neuroradiol 2011; 32:709-13

14. Viswanathan A, Guichard JP, Gschwendtner A, et al. Blood pressure and haemoglobin Alc are associated with microhaemorrhage in CADASIL: a two-centre cohort study. Brain 2006;129:2375-83

15. Cho S, Jones D, Reddick WE, et al. Establishing norms for age-related changes in proton T1 of human brain tissue in vivo. Magn Reson Imaging 1997;15:1133-43

16. Iannucci G, Dichgans M, Rovaris M, et al. Correlations between clinical findings and magnetization transfer imaging metrics of tissue damage in individuals with cerebral autosomal dominant arteriopathy with subcortical infarcts and leukoencephalopathy. Stroke 2001;32:643-48

17. Chabriat H, Pappata S, Poupon C, et al. Clinical severity in CADASIL related to ultrastructural damage in white matter: in vivo study with diffusion tensor MRI. Stroke 1999;30:2637-43

18. Yao M, Jouvent E, During M, et al. Extensive white matter hyperintensities may increase brain volume in cerebral autosomal-dominant arteriopathy with subcortical infarcts and leukoencephalopathy. Stroke 2012;43:3252-57

19. Kong L, Herold C, Stieltjes B, et al. Reduced gray to white matter tissue intensity contrast in schizophrenia. PLoS One 2012;7:e37016

20. Salat DH, Lee SY, van der Kouwe AJ, et al. Age-associated alterations in cortical gray and white matter signal intensity and gray to white matter contrast. Neuroimage 2009;48:21-28

21. Salat DH, Chen JJ, van der Kouwe AJ, et al. Hippocampal degeneration is associated with temporal and limbic gray matter/ white matter tissue contrast in Alzheimer's disease. Neuroimage 2011;54:1795-802 